\title{
Intervención educativa para el alumnado con rotacismo junto con el grupo clase
}

\section{Educational programme for students with rhotacism along with the whole class}

\author{
Eugenia Díaz-Caneiro, Marta Gestal-Couso \\ Universidade da Coruña
}

\begin{abstract}
Resumen
Los trastornos del habla conllevan dificultades para comunicarse, limitaciones en el aprendizaje y temores que condicionan el desarrollo social. Estamos ante un estudio de caso único con un niño de 6 años que presentaba una dificultad del habla sin haber sido diagnosticado. Nuestros objetivos son promover el desarrollo lingüístico del alumnado y eliminar barreras segregadoras en el aula. Valorados los logros de la intervención, podemos concluir que las actividades de estimulación de las habilidades fonológicas han ayudado a compensar dichas dificultades y permiten mejorar el rendimiento académico. Además, el ambiente en el aula se ha convertido en integrador e inclusivo.

Palabras clave: evaluación del lenguaje, fonología, inclusión, interdisciplinaridad, lenguaje oral
\end{abstract}

\begin{abstract}
Speech disorders involve difficulties for communication, learning and social development. This project deals with a case study with a six-year-old child who had a speech disorder but had not been identified yet. Our aims are to promote students' linguistic development and to avoid segregation in the classroom. After assessing the intervention, we can state that the activities for stimulating the phonologic skills have helped to overcome previous difficulties and to improve the academic efficiency. Furthermore, the atmosphere has become integrating and inclusive.
\end{abstract}

Keywords: cross-curricular, inclusion, language assessment, oral language, phonology.

\section{Introducción}

En la infancia hay una progresiva adquisición de funciones importantes, como pueden ser el control postural, la autonomía de desplazamiento, la interacción social o el lenguaje (Conde, Conde, Bartolomé y Quiroz, 2009). Sin embargo, es muy frecuente que surjan alteraciones del lenguaje oral, siendo los trastornos más comunes los retrasos en el desarrollo del lenguaje, la tartamudez y las dislalias (Quintana, Aguilar, Lantigua, tasé, Calixto y Contreras, 2013). La dificultad puede implicar a todos, uno o algunos de los componentes fonológico, morfológico, semántico, sintáctico o pragmático del sistema lingüístico (Barrachina, Aguado, Cardona y Sanz, 2013) y tanto de forma oral como escrita (Schwartz, 2009).

El objeto de nuestro estudio es un caso de dislalia, que popularmente designaban cualquier problema de habla en el que estuviese afectada la articulación, la percepción o la representación mental de los sonidos de la lengua (Gallego, 2013). Sin embargo, hace referencia a los trastornos del lenguaje que no se deben a lesiones neurológicas evidentes (disartrias), malformaciones anatómicas (disglosias), sordera (dislalia audiógena) ni déficit intelectual, sino que la alteración del desarrollo del lenguaje se restringe a la articulación (Ferreira, Keske y Alves, 2010).

En nuestro caso, se trata de una dislalia fonética, que altera la ejecución de los sonidos por los articuladores, pero no existe un problema de discriminación auditiva, por lo que los errores son constantes y afectan a sonidos concretos (Gallego y Rodríguez, 2009). Éste sonido específico será el fonema $/ r /$, que se define como rotacismo, es decir, que tiene dificultad para su pronunciación ya que requiere una vibración de la lengua contra el paladar y una posición de la boca en la cual se dificulta el aprendizaje por observación (Redondo, 2008).

Nuestra labor consiste, por consiguiente, en detectar si estas dificultades se deben a un mal funcionamiento de los órganos que intervienen en el habla, a posiciones incorrectas de labios o lengua, incorrecta respiración, falta de aire, escasa movilidad de labios, lengua o paladar (Carrasco, García y Hervas, 2015). Si no existe una detección temprana, tiene una alta probabilidad de persistencia que implica unos efectos devastadores tanto para el rendimiento académico como para el desarrollo socioemocional (Conti-Ramsden, St Clair, Pickles y Durkin, 2012). La intervención no solo consigue que se produzca una mejoría directa, sino que también beneficia el incremento en otros niveles como pueden ser la atención, percepción, motor o memoria (Robles, Sánchez y Valencia, 2015).

Existen numerosas aproximaciones en los programas de intervención que en los últimos años se han aplicado con sujetos con trastornos del lenguaje (Acosta, 2012). Algunos mejoran la lectura inicial en este alumnado, a nivel fonológico y de desarrollo narrativo (Coloma, Pavez, Peñaloza, Araya y Palma, 2012), mientras que otros se centran en la comprensión y expresión del lenguaje oral (Acosta, 2014).

Todavía no hay acuerdo acerca de qué procedimientos utilizar, con qué intensidad y frecuencia, qué tipos de actividades, cuáles son los agentes que deben implicarse o en qué contextos deben implementarse los programas 
de intervención (Acosta, Moreno y Axpe, 2012). Sin embargo, lo que debemos tener en cuenta es que el alumnado con rotacismo obtiene puntuaciones mucho más bajas que sus iguales en el desarrollo del lenguaje, pero están dentro de los límites normales en los aspectos cognitivos no-verbales y no presentan déficit sensorial, emocional ni neurológico (Washington, 2013), por lo que consideramos muy beneficioso realizar una intervención individualizada y una conjunto de actividades grupales con el grupo-clase que refuercen nuestro programa de intervención.

\section{Método}

En el estudio participó un niño de 6 años de edad, que presentaba una dificultad del habla sin haber sido diagnosticado previamente. El trabajo se desarrolló a lo largo de tres fases. La primera de ellas consistió en una evaluación inicial donde diseñamos una serie de actividades para reconocer el tipo de trastorno del habla que presentaba. Una vez averiguado que se trataba de un caso de dislalia funcional, más concretamente de rotacismo, hemos diseñado un plan de intervención para estimular el fonema / $\mathrm{r} /$ en sus diferentes posiciones. Finalmente, la tercera fase ha consistido en la elaboración de una evaluación final con el fin de valorar los resultados alcanzados por el alumno.

Nuestra meta fue facilitar a la familia una guía en la cual apoyarse e informarse acerca de este fenómeno y conseguir la mejora del niño a la hora de pronunciar el fonema /r/ en posición inicial, medial y final. Nuestra propuesta de ejercicios es aplicable en todos los ámbitos en los que se encuentra el alumno busca acelerar los avances y la evolución para conseguir que el niño pronuncie adecuadamente a la mayor brevedad posible.

\section{Procedimiento}

Hemos realizado una unidad didáctica para llevar a cabo con el participante con el fin de seguir una atención personalizada basada en actividades lúdicas donde el alumno es el foco de aprendizaje. La unidad didáctica ha sido titulada "'Erre que erre". Hemos elegido este nombre, por dos razones: 'Erre que erre' es una expresión conocida en nuestro país a la que atribuimos el significado de insistencia y terquedad. Tiene gran relación con nuestro objetivo: trabajar diariamente con ejercicios propuestos, consiguiendo que con dedicación e interés de dicho alumno logre la correcta articulación del fonema / r /. La otra razón, evidentemente, es porque este espacio va a estar dedicado a trabajar exclusivamente el fonema $/ \mathrm{r} /$.

La unidad cuenta con 13 sesiones que se trabajaron con ese alumno en las clases impartidas por otros docentes, así nuestra carga lectiva nos permitió estar disponibles en esas franjas horarias y así poder atenderlo personalmente como tutores. 11 sesiones fueron de temas genéricos relacionadas con la materia de lengua castellana y 2 sesiones de temáticas festivas del trimestre, realizando una para el magosto y otra para navidad.

La unidad didáctica se desarrolló a lo largo de tres fases. La primera de ellas consistió en una evaluación inicial donde diseñamos una serie de actividades para reconocer el tipo de trastorno del habla presentaba el niño, la segunda dedicada a ejercicios y gimnasia con la boca del alumno delante de un espejo y la última diferentes actividades donde se trabaja el fonema / $\mathrm{r}$ / en posición inicial, medial y final y también los correspondientes sinfones /br/ /dr/, etc.

Los contenidos que trabajaremos son: control del soplo; discriminación auditiva y visual de los fonemas /r/, /d/, /l/, / $\overline{\mathrm{r}} /$; discriminación auditiva y visual de los sinfones /br/ y /dr/; el fonema /r/: la lengua y su correcta posición; el fonema /l/; el fonema /d/ y la memoria.

Entre las actividades, debemos destacar: repaso colocación de la lengua delante del espejo; ejercitado de los músculos de la boca; soplo (pipas con pelota, pompas de jabón, circuito con pelota y pajita, dibujo con acuarelas y pajita); fichas de los fonemas / r / y /d/; la oca de la R; fichas de los fonemas / $\mathrm{r} / \mathrm{y} / \mathrm{l} /$; fichas de los fonemas / $\mathrm{r} / \mathrm{y} / \overline{\mathrm{r}} /$; sinfones $/ \mathrm{br} / \mathrm{y} / \mathrm{dr} /$; repetición de palabras; juegos de parejas y cuentos sobre gimnasia con la boca.

\section{Resultados}

Teniendo en cuenta los criterios de evaluación y los estándares de aprendizaje recogidos en la ley actual, al tratarse de un trabajo individualizado donde solo hay un alumno, basaremos la evaluación en la mera observación del progreso de dicho alumno, tanto en las sesiones individuales con la tutora, como la actuación e inclusión de lo aprendido con el resto del aula.

Los datos obtenidos muestran una mejora en el fonema / r / que fue objeto de intervención. Además, hemos descubierto que las actividades enfocadas al desarrollo de habilidades fonológicas interfieren positivamente no sólo en el aprendizaje de la totalidad de las materias curriculares sino también en el desarrollo personal del alumno, ya que es capaz de hacerle frente a sus barreras de aprendizaje. Valorados los logros de la intervención, podemos afirmar que las actividades de estimulación de las habilidades fonológicas en el alumnado con trastornos del habla ayudan a compensar dichas dificultades y permiten mejorar el rendimiento académico.

Asimismo, hemos detectado actitudes segregadoras por parte de los compañeros del alumno que debían ser eliminadas. Por consiguiente, hemos diseñado una propuesta didáctica que nos permite trabajar con la totalidad del grupo la dificultad de dicho alumno y fomentar valores éticos que permitan, por una parte, que nuestro sujeto acepte su situación y, por la otra, que el resto de compañeros sean conscientes de la diversidad existente en el aula.

Hora de lectura. Leeremos en la hora de lectura el libro 'Un sueño extraño", con todo el aula. Primeramente trabajaremos dicho cuento en las clases individuales con el niño que presenta la dificultad. Esto es así ya que el libro trata de trabajar las praxias y realizar gimnasia con la boca. Lo que pretendemos con esto es que el niño ya domine alguno de los ejercicios que este presenta ya que al resto de sus compañeros les resultará más fácil debido a que ellos tienen un mayor de control de la lengua que el niño que presenta rotacismo. Creemos 
que este cuento puede dar mucho juego ya que los niños piensan que están jugando y en realidad están haciendo ejercicio con la boca, lo que permite un mayor control de la lengua.

Lengua castellana. Cuando tratemos en la materia de lengua la letra y el fonema /r/ entregaremos al alumnado diferentes fichas en las que tengan que distinguir los siguientes fonemas: / r / y / / / / r / y / d /; / r/ y / ${ }^{-} \mathrm{r} / \mathrm{y}$ Sinfones / br / / dr / / tr / / fr /.

Ciencias naturales. Por grupos de cuatro o cinco personas tendrán que buscar 3 animales que contenga o empiece por la letra / r /. A continuación por grupos tendrán que representar uno de los tres animales que eligieron, otro debe ser adivinado por el juego del ahorcado y el tercero dando pistas, como por ejemplo: tierra, reptil, verde, pequeño, con patas, etc. Cada grupo tendrá que comentarnos que animales eligieron para que de esta forma no se repita ningún animal en los diferentes grupos.

Lengua gallega. En el segundo o tercer trimestre, entre todos llevaremos a cabo un cuento relacionado con la primavera y las flores. Intentaremos buscar las máximas palabras que contengan / r/.

Música. Imitamos sonidos: croac, croac (rana); brrrr (moto arrancando), etc.

Valores. Trabajamos en el aula con la ayuda del cuento Emocionario los valores como el: respeto, igualdad, tolerancia...

\section{Conclusión}

Los humanos comenzamos a adquirir el lenguaje en nuestro entorno más cercano para después continuar desarrollándolo cuando entramos en el sistema educativo. Sin embargo, el ritmo del desarrollo lingüístico en el alumnado varía considerablemente, por lo que es en la escuela donde prevalecen los problemas lingüísticos.

Para nosotros el proceso de enseñanza - aprendizaje del lenguaje oral es más complicado que el de una materia curricular ya que el alumno lo necesitará no solo para su desarrollo personal fuera de la escuela, sino que también lo utilizará para alcanzar los objetivos de aprendizaje establecidos en las demás materias. Además, el contexto sociocultural del niño jugará un papel muy importante que debemos tener en cuenta como maestros.

Con este proyecto hemos buscado promover el desarrollo lingüístico del alumno que presenta trastornos del habla y eliminar barreras segregadoras en el aula. La intervención individual ha demostrado que el participante ha sido capaz de mejorar sus dificultades, no solo con el fonema / $\mathrm{r} /$, sino también ha progresado en su nivel de autoestima y consideración personal al darse cuenta de que es capaz de superarse cada día un poco más.

Dada la interdisciplinaridad de nuestras actividades propuestas, los alumnos serán capaces de descubrir por ellos mismos las fortalezas y debilidades de cada uno. Así, nuestro alumno con rotacismo restará importancia a su dificultad de aprendizaje al darse cuenta de que sus compañeros también presentan dificultad en otros ámbitos de otras materias curriculares. Esto fomenta valores educativos como el respeto, igualdad, tolerancia o aprendizaje colaborativo de inteligencias múltiples.

Para la intervención, hemos considerado extremadamente necesario el trabajo conjunto de maestros, psicopedagogos, lingüistas y psicólogos, con el fin de ayudar a los estudiantes de una forma conjunta y global. Parte de ellos se han visto involucrados en este caso y los resultados serían todavía más sobresalientes si contásemos con mayor presencia. Para concluir, nos gustaría animar a todos estos profesionales a trabajar conjuntamente para la mejora del proceso de enseñanzaaprendizaje, ya que sólo aunando esfuerzos podemos conseguir que aquellos alumnos con dificultades sean capaces de superarlas y que desaparezcan las barreras segregadoras en las aulas.

\section{Referencias}

Acosta, V. (2012). Algunos retos y propuestas en la conceptualización, evaluación e intervención del Trastorno Específico del Lenguaje. Revista Chilena de Fonoaudiología, 11, 23-36.

Acosta, V. (2014). Efectos de un programa de intervención en el lenguaje en alumnado con Trastorno Específico del Lenguaje. Revista de investigación en Educación, 12 (1), 92-103.

Acosta, V., Moreno, A. y Axpe, A. (2012). La acción inclusiva para la mejora de habilidades de lenguaje oral y de lectura inicial en niños con Trastorno Específico del Lenguaje. Revista de Educación, 359, 332-356.

Barrachina, L., Aguado, G., Cardona, M. y Sanz, M. (2013). El trastorno específico del lenguaje diagnóstico e intervención. Editorial UOC. Barcelona.

Carrasco, A., Garcia, R. y Hervas, M. (2015). Revisión de programas de intervención dirigidos a alumnos de infantil y primaria con trastorno específico del lenguaje. ReiDoCrea, 4, 156-161.

Coloma, C., Pavez, M., Peñaloza, C., Araya, C., y Palma, S. (2012). Desempeño lector y narrativo en escolares con Trastorno Específico del Lenguaje. Onomázein, 26, 351-375.

Conde, P., Conde, M., Bartolomé, M., y Quiróz, P. (2009). Perfiles neuropsicológicos asociados a los problemas del lenguaje oral infantil. Revista de Neurología, 48, 32-40.

Conti-Ramsden, G., St Clair, M., Pickles, A y Durkin, K (2012). Developmental trajectories of verbal and nonverbal skills in individuals with a history of specific language impairment: from childhood to adolescence. Journal of Speech, Language, and Hearing Research, 55 (2), 1716-1735

Gallego, J. (2013). Los trastornos de lenguaje en el niño. Estudios de caso. Sevilla: Eduforma

Gallego, C, Rodríguez-Santos, F. (2009) Trastornos específicos del lenguaje. En: AEPap (ed.) Curso de Actualización Pediatría (239-248) Madrid: Exlibris Ediciones.

Ferreira, G., Keske, M. y Alves, M. (2010). Estudo do papel do contexto linguístico no tratamento do desvio fonológico. Revista da Sociedade Brasileira de Fonoaudiologia, 15 (1), 96-102 
Quintana, D., Aguilar, L., Lantigua, P., Tasé, D., Calixto, Y. y Contreras, J. (2013). Características electroencefalográficas de niños con trastornos en el desarrollo del lenguaje con y sin histidinemia. Revista Cubana de Neurología y Neurocirugía, 3, 117- 121.

Redondo, A. (2008) Trastornos del Lenguaje. Pediatría Integral, 12 (9), 859-872

Robles, M., Sánchez, D., Valencia, N. (2015). Evaluación del potencial de aprendizaje en niños con Trastorno Específico del Lenguaje. Revista de Psicología clínica con niños y adolescentes, 2(1) 19-24.

Schwartz, R. (2009). Specific language impairment. En R. Schwartz (Ed.). Handbook of child and language disorders (3-43). New York: Pyschology Press.

Washington, K. (2013). The association between expressive grammar intervention and social and emergent literacy Outcomes for Preschoolers With SLI. American Journal of Speech-Language Pathology, 22 (1), 113-125. 